Vol. 44 (1991) [33-43]

\title{
THE PATTERNS OF THE ISONEMAL TWO-COLOUR TWO-WAY TWO-FOLD FABRICS
}

\author{
J.A. Hoskins and R.S.D. Thomas
}

\begin{abstract}
This paper connects the literature on weaving with that on coloured tilings of the plane. It sets the many published designs of woven cloth in the context of $(2,2,2)$ fabrics, that is, those in which strands of two colours run in two perpendicular directions to cover the plane doubly almost everywhere. At the same time, it connects with the interesting topological question of when $a$ weave hangs together. The main result is that isonemal $(2,2,2)$-fabrics can be made from isonemal uncoloured fabrics either by colouring them in the conventional way (black warp, white weft), or by colouring warp and weft in thin or thick stripes to give the visual appearance of conventionally coloured isonemal pre-fabrics that fall apart, and in no other way. Such visual appearance can always be realised by the striping of an isonemal fabric, but not uniquely. A figure showing the fifty isonemal $(2,2,2)$-fabrics of exact orders up to sixteen coloured by striping is included.
\end{abstract}

\section{INTRODUCTION WITHOUT COLOUR}

This paper is meant to be a contribution to the mathematical literature of weaving that has been accumulating for the ten years from the publication of [8] (the best introduction to the subject) to [7], which is the most up-to-date treatment known to us. Some of the terminology introduced over this period-by the authors among others-is unhelpful; some of it we shall simply ignore, but we shall indicate terms not in the above references or different use of those that are. Most of the literature is intended to refer to mathematical models of real or possible products of the ancient craft of weaving; that is the intention of this paper, which will be restricted to two-way weaves (in which the strands run in two directions at right angles to each other-warp or vertical and weft or horizontal) and to two-fold weaves (in which there are never more than two strands crossing each other) that are periodic (defined below). Because these three restrictions are understood at the outset, we shall not continually mention them, as in the following definition. We call a prefabric a planar arrangement of infinite strands into two layers such that, at every point of the plane that does not lie on the

Received 1 October 1990

The authors gratefully acknowledge the assistance of the Natural Sciences and Engineering Research Council of Canada.

Copyright Clearance Centre, Inc. Serial-fee code: 0004-9729/91 \$A2.00+0.00. 
boundary of one of the strands, one or the other of the vertical and horizontal strands is uppermost. For a more formal treatment of the ranking of the strands, as well as for the possibilities of $n$-way and $n$-fold prefabrics, [7] (which some of the above wording imitates) should be consulted. It is of some importance that the mathematical notion of prefabric does not include that the physical structure modelled will hang together, as does most cloth; the matter of hanging together and its opposite falling apart has been one of consistent interest in this theory $[8,1,10,6,4,2]$. We further this discussion.

A symmetry of a prefabric is defined in [7] to be any isometry of the plane of the strands that maps each strand of the prefabric $P$ onto a strand of $P$ and either preserves the rankings at each point or else reverses all the rankings. A prefabric is called periodic if the normal subgroup of its symmetry group consisting of isometries that preserve rankings contains translations in at least two nonparallel directions. As we remarked at the outset, our prefabrics are assumed to be periodic. A prefabric (understood to be two-way, two-fold, and periodic) is called a fabric if it hangs together. A prefabric is called isonemal if its symmetry group is transitive on its strands. Since an isonemal prefabric has symmetries taking warp strands to weft strands, the symmetry group contains equal translations of $n$ strands in both horizontal and vertical directions; the smallest such integer $n$ we shall call the exact order of the prefabric, and call its multiples orders. The term 'order' was introduced in [8] with this meaning. It is a convention of the weaver's craft to represent the ranking of strands of a prefabric in a diagram called a design that is precisely the appearance of the prefabric if its warp strands are coloured black and its weft strands are coloured white. The structure of a prefabric of exact order $n$ is fully represented by a square diagram consisting of $n^{2}$ squares coloured black or white, and virtually all the weaving literature follows this convention. (That $[\mathbf{1 0}]$ is an exception allowed the misprinted first two rows of the first design in Table 1 of [10] to go uncorrected.) Much of the visual appeal of the weaving literature depends on this convention, which makes the structure much more apparent than it is in actual cloth woven of strands of a single colour. As soon as one wishes to discuss the colouring of the warp and weft strands, however, the convention entails much possibility of confusion. (See [9], Section 8.8.)

\section{INTRODUCTION OF COLOUR}

This paper is also meant to be a contribution to the literature of colour symmetry; Escher's jibe about scientific 'colourlessness' [5] is no longer apt thirty years later. We shall refer to the colouring of the strands that results in the appearance displayed in the conventional design of an uncoloured prefabric (warp and weft uniform and different) as the normal colouring. A two-coloured prefabric will be called isonemal if

1. the uncoloured prefabric is isonemal and 
2. the strands are perfectly two-coloured; that is, every symmetry of the uncoloured prefabric induces a permutation of the colours (the identity or the interchange of the colours of all of the strands).

Colourings of the strands of a prefabric of exact order $n$ in any way at all with vertical and horizontal period $n$ will result in a visual appearance representable by a square diagram consisting of $n^{2}$ squares each coloured with the colour of the strand uppermost there. We shall refer to this appearance as the pattern of the coloured prefabric. Accordingly the design of a prefabric is equivalent to the pattern of its normal colouring. Any such $n^{2}$ squares are the design of some prefabric but not necessarily the pattern of a fabric. The designs of the isonemal prefabrics of exact orders up to sixteen that fall apart are illustrated in Figure 1.

It should be noted that the area of a fundamental block or period parallelogram of a pattern (which generates it by translations) can be smaller than the square of the exact order of the prefabric. In the first entry in Figure 1.1, 4-1-1*, it is half the area. In other cases it is less, and in the case of some sponge weaves (see [7] or [8]) it is a small fraction. This discrepancy suggests to the authors the avoidance of the term 'period' for order. It can further be noted that what the eye fastens on tends to be a motif that generates the pattern by isometries and colour interchange, for example a zig zag of a single colour in the first, fourth, and fifth entries in Figure 1. Except in very few entries in Figure 1, this motif is itself infinite and periodic with a period totally unrelated to order. The exceptions are interesting, expecially how few of them there are only 4-1-2*, 4-1-3*, 8-9-1* (omitted from [6]), 16-2193-1*, and 16-2193-2*.

The patterns of exact order sixteen for the most part extend families of patterns at lower orders. The family of ornamented doubled zigzags, which begins with $8-11-9$ (see Figure 2), is expanded with $153-1^{*}, 153-2^{*}, 291-1^{*}, 291-2^{*}, 291-3^{*}, 531-3^{*}, 531-$ $4^{*}, 561-2^{*}$. The family of finite motif within an infinite motif, which begins in the simplest possible way with $8-5-3^{*}$, begins containing interesting motifs with $291-4^{*}$, $531-1^{*}$, and $561-1^{*}$. A wholly new family is that which mixes finite motifs, namely that of $8-9-1^{*}$ with that of $4-1-2^{*}$ in $2193-1^{*}$ and with that of $4-1-3^{*}$ in $2193-2^{*}$. Attention should also be drawn to the interestingly different pair $1093-3^{*}$ and $1105-2^{*}$.

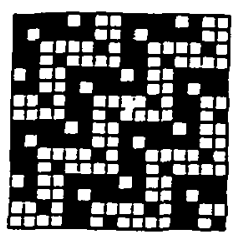

Figure 2. The simplest of the ornamented doubled zigzags, found in [10], numbered 8-11-9 in [6]. 


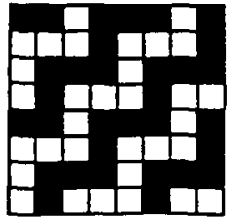

4-1-1*

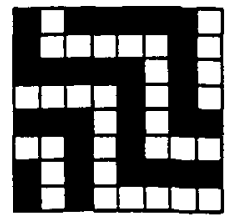

8-5-1*

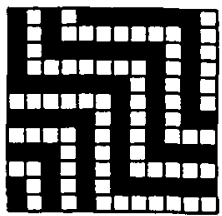

12-21.1*

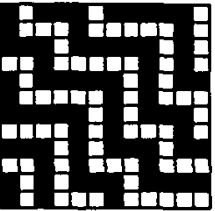

$12-69-1^{*}$

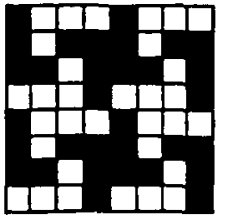

4-1-2*

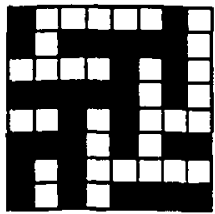

8-5-2*

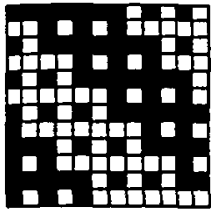

$12-21-2^{4}$

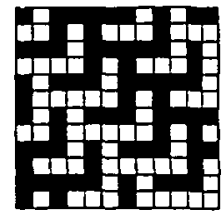

$12-69-2^{*}$

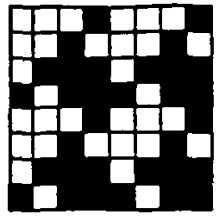

4-1-3*

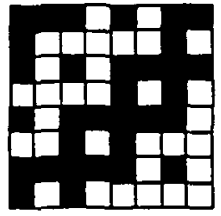

8-5-3*

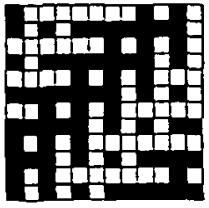

$12-21-3^{*}$

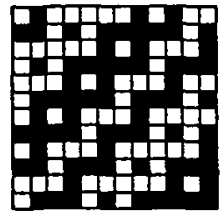

$12-69-3^{*}$

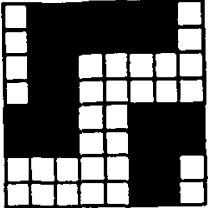

8-3-1"

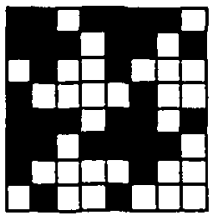

8-9-1*

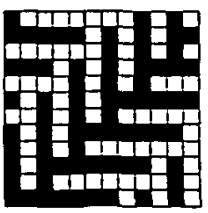

$12-21-4^{*}$

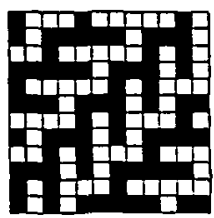

$12-69-4^{*}$

Figure 1.1

\section{Colouring a weave}

LEMMA 1. The normally coloured isonemal fabrics are isonemal two-colour fabrics.

This fact, which we regard as obvious, has been implicit in the mathematical weaving literature stimulated by [8]. Fabric designs are the patterns of perfect two-colourings of the fabrics. Normal colouring is not, however, the only way that such fabrics can be two-coloured perfectly. We must consider how strands can be non-normally two- 


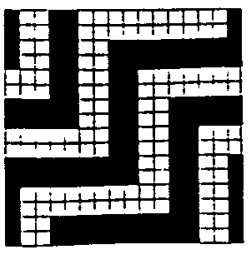

16-51-1

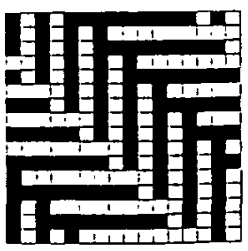

$16-85-2^{*}$

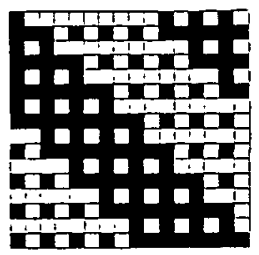

$16-85-5^{*}$

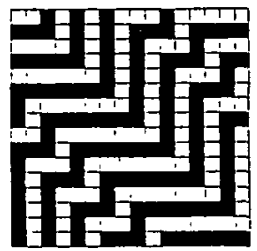

16-277-1"

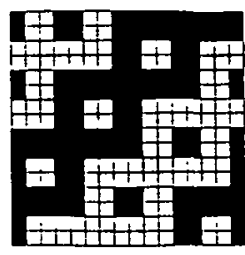

16-51-2"

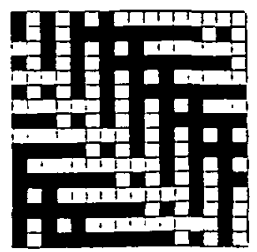

$16-85-3^{*}$

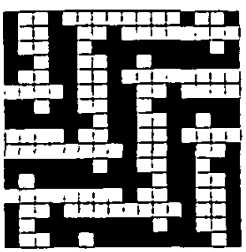

16-153-1"

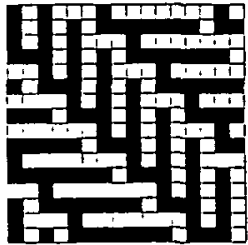

16-277-2

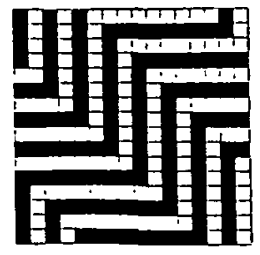

$16-85-1^{*}$

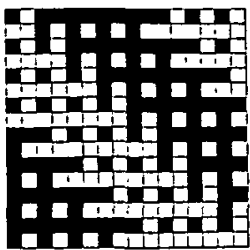

$16-85-4^{*}$

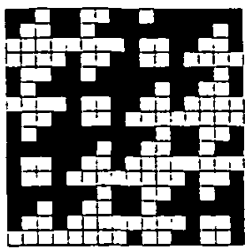

16-153-2

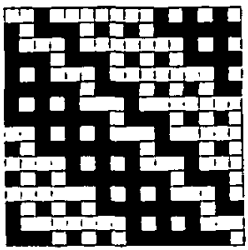

$16-277-3^{4}$

Figure 1.2

coloured perfectly. Each strand can be taken to its two adjacent strands by symmetries (possibly inverses of each other modulo translation) called construction [10] or transition [11] rules (so called because they determine the construction of the whole prefabric from a single strand by transitions to adjacent strands). Whether the rules for the two transitions are inverses or not, they may both induce colour interchange and the strands are alternately coloured or they may induce different colour permutations, that is, one 


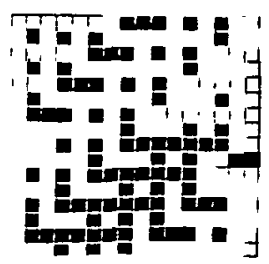

$16-277-4$

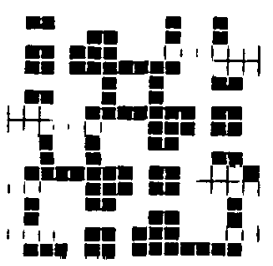

$16-291-3^{*}$

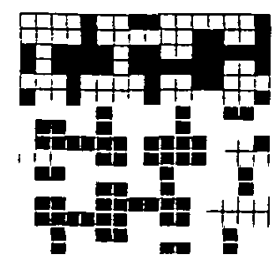

16-531-2*

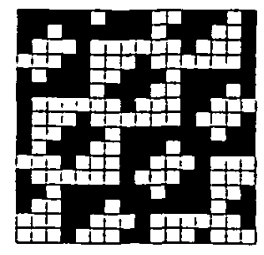

16-561-1*

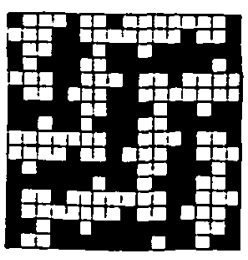

$16-291-1 *$

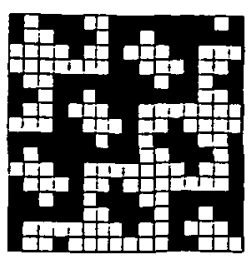

$16-291-4^{*}$

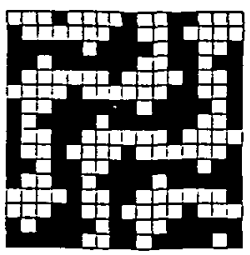

$16-531-3^{*}$

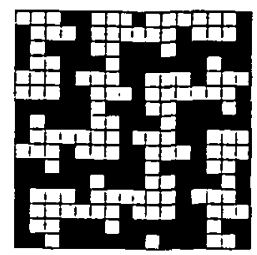

$16-561-2^{4}$

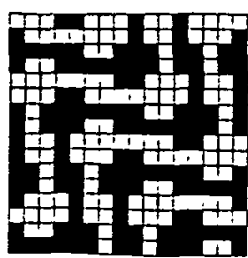

16-291-2*

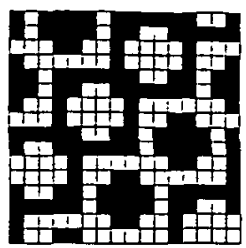

16-531-1*

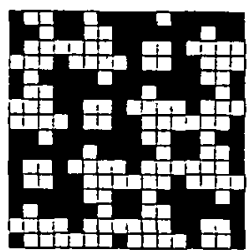

$16-531-4$

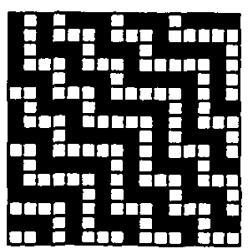

16-1093-1*

Figure 1.3

the identity and the other interchange. In the latter case the colours of the strands alternate in pairs. The same is true of both warp and weft strands because of isonemality, so that the warp and weft are striped either thinly or thickly. We have proved the following lemma.

LEMMA 2. Isonemal two-colour fabrics not normally coloured must have thinly 


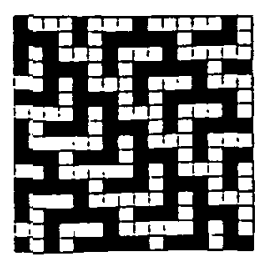

$16-1093-2$

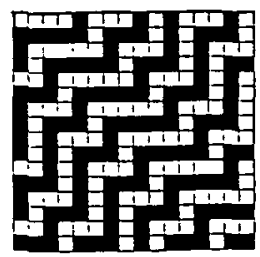

$16-1105-1 *$

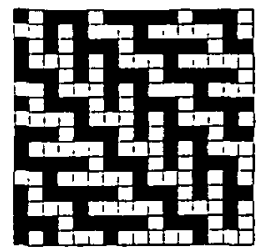

16-1105-4*

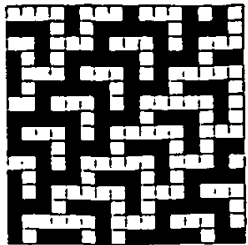

16-1093-3"

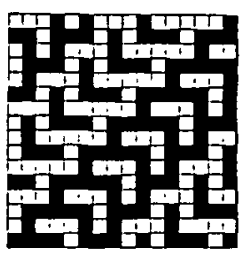

16-1105-2*

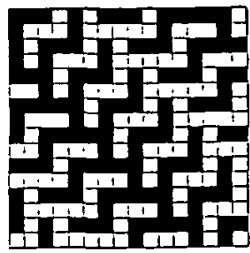

16-1105-5*

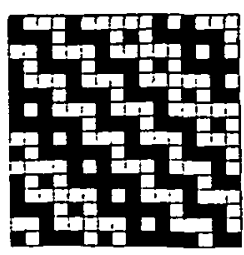

$16-1093-4$

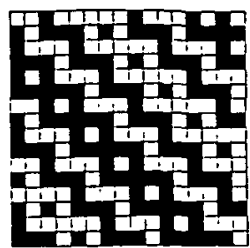

$16-1105-3^{*}$

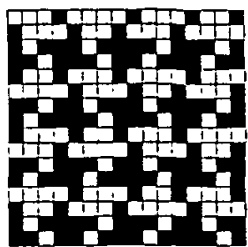

$16-2193-1$ *

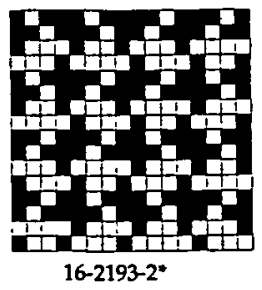

Figure 1.4

Figure 1. The designs of the isonemal prefabrics of exact orders up to sixteen that fall apart. The numerical labels are explained at the end of Section 3.

or thickly striped warp and weft.

It should be noted that striping warp and weft is a standard weaving device not the invention of the authors. The actual alternation of colours forced upon weavers 
to produce some of the most attractive patterns that there are (including the simplest houndstooth, 4-1-2* in Figure 1.1) renders unsuitable the term 'colour alternation' used in the earlier mathematical literature on weaving in a wholly colourless (structural) sense; it is superseded by the language of genera introduced in [6]. Isonemality need not be assumed in the following lemma.

LеммA 3. The pattern of a two-colour fabric made from striped warp and weft cannot be a (normally coloured) fabric design; it must be the design of a prefabric that falls apart.

Proof: Take the $2 n \times 2 n$ pattern of a two-colour fabric, say black and white, made from thinly or thickly striped warp and weft, and consider this pattern to be normally coloured fabric. This will cause the pattern and the design to be equivalent and will result in a lattice of black squares, where a black warp strand crosses a black weft strand, and a lattice of white squares, where a white warp strand crosses a white weft strand which are independent of the interlacement structure. The colour that appears at the remaining intersections is wholly dependent on the interlacement structure. The warp and weft strands can obviously be permuted so as to produce a pattern (and design) with an $n \times n$ black square in the upper left corner and an $n \times n$ white square in the lower right corner, a necessary and sufficient condition for this design to be that of a prefabric that falls apart [12].

Corollary. Isonemal two-colour fabric patterns that are made with striped warp and weft are of exact order a multiple of four and are the designs of the isonemal prefabrics of genus $I I, I V$, or $V$ with one quarter of the squares in half the rows black.

ProOF: The non-trivial isonemal prefabrics that fall apart were determined in [10] to be of exact order a multiple of four, and of genus II, IV, or V (that is, having at least one transition rule involving reversal of ranking) such that their designs have exactly one quarter of the squares in half the rows black. An elegant proof has since appeared in [2].

THEOREM 1. Isonemal two-colour fabrics can be made from isonemal fabrics either by colouring them normally or so that they have the patterns of normally coloured isonemal prefabrics that fall apart if that colouring is possible.

Theorem 1 is proved by Lemmas 1, 2 and 3. An example of striping to produce a non-normally two-coloured pattern is shown in Figure 3. It is based on a twill (all twills are isonemal) of exact order eight which, depending on how it is thinly striped, has the pattern of the prefabric $8-5-1^{*}$ of exact order eight or 4-1-1* of exact order four, showing that the order of a pattern can be a proper factor of the order of the underlying weave.

Isonemal two-colour fabric patterns can be divided on account of Theorem 1 into 

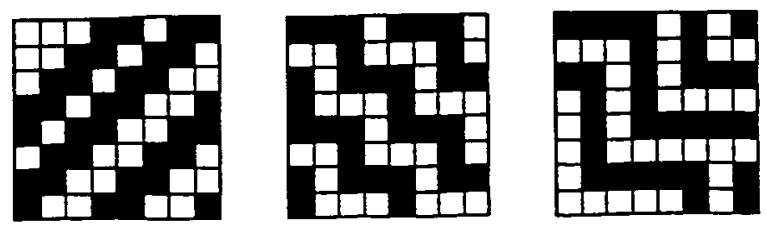

Figure 3. The design of a twill of exact order eight and its colouring by thin striping to produce the prefabric patterns 4-1-1* and 8-5-1*.

six genera, five corresponding to the designs of the normally coloured fabrics of the overlapping genera I to $\mathrm{V}[6]$ and the distinct sixth being the designs of the isonemal prefabrics that fall apart. The patterns of genus VI in Figure 1 are identified like those in Figure 5 of [6] illustrating the designs of isonemal fabrics that are not twills: exact order - binary index - serial number (with an asterisk to avoid duplication with the serial numbers in [6]). The binary index is the decimal expression of the minimum (under cyclic permutations of the row, reversal of order, and reversal of black and white) of the $n$-digit binary number obtained from a row by interpreting each black square as 1 and each white square as 0 .

\section{What WEAVE TO COLOUR}

The example of Figure 4 indicates that the pattern 4-1-1* can be produced from a twill with the design sequence black, black, white, white or black, white, white, white or their concatenation, just as (in Figure 3) from the sequence black, white, white, white or black, black, white, black, (not really different) or their concatenation.
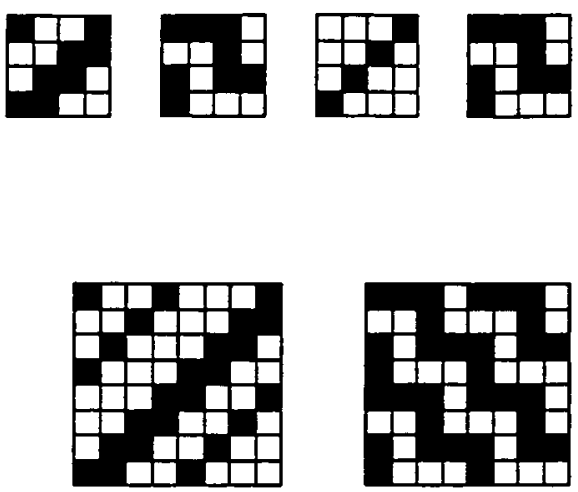

Figure 4. The designs of two twills of exact order four and one of exact order eight, all of which can be thinly striped to produce the pattern 4-1-1*. 
There is a great deal of freedom in the choice of the isonemal fabric from which to produce an isonemal two-colour fabric if it is not normally coloured. There is, however, a simple choice, and our second theorem shows that it is always available.

TheOREM 2. An isonemal two-colour fabric not normally coloured can always be produced from an isonemal fabric determined by a simple algorithm.

Proof: In order to specify the simple algorithm, we need to divide the squares of the black and white pattern into two classes, those where rows and columns of predominantly the same colour cross, which we call uninteresting, and those where rows and columns of predominantly different colours cross, which we call interesting. To produce the design of an underlying isonemal fabric from the pattern, colour the uninteresting squares white and colour the interesting squares according to the normal rule, that is, warp uppermost black and weft uppermost white. Which strand is uppermost must be determined by comparison of the colour of the square in the pattern with the (different) colours of the corresponding warp and weft. The algorithm is certainly simple, and it does produce a prefabric design. We must show that the prefabric is isonemal and hangs together.

The first thing to note is that the uninteresting and interesting squares form a chequerboard with one another, the size of the squares depending on whether the pattern is thinly or thickly striped. By the isonemality of the pattern, the symmetries of the pattern take rows and columns of predominantly the same colour to rows and columns of predominantly either the same colour or (both) of predominantly the other colour. Accordingly, interesting squares go to interesting squares and uninteresting squares go to uninteresting squares in all symmetries of the pattern. The uninteresting squares have all been made white, and so all the symmetries of the pattern preserve their colouring. For the isonemality of the pattern there must be a symmetry to take any strand to any strand, perhaps reversing the colours of all the strands. The same isometries still take any strand to any strand in the design; the ranking of the interesting squares was carefully preserved by the algorithm. Colour reversal in a pattern symmetry is necessary because strands of predominantly one colour are being mapped by the isometry to strands of predominantly the other colour. In the design there is no colour to be reversed, and so the symmetries of the design are the symmetries of the pattern except that, in the design symmetries, there are no colour reversals, just isometries. These symmetries are sufficient for isonemality of the design.

The second thing to note is that, because every row and column has been constructed to be at least one half white, the isonemal prefabric designed fails to be of a kind that can fall apart (as specified in the proof of the Corollary to Lemma 3 ). 


\section{REFERENCES}

[1] C.R.J. Clapham, 'When a fabric hangs together', Bull. London Math. Soc. 12 (1980), 161-164.

[2] C.R.J. Clapham, 'The bipartite tournament associated with a fabric', Discrete Math. 57 (1985), 195-197.

[3] M.C. Escher: Art and science, Editors H.S.M. Coxeter et al. (North-Holland, Amsterdam, 1986).

[4] T.C. Enns, 'An efficient algorithm determining when a fabric hangs together', Geom. Dedicata 15 (1984), 259-260.

[5] M.C. Escher, Letter to Arthur Escher of 5 July 1959, quoted in [13, p.83].

[6] B. Grünbaum and G.C. Shephard, 'A catalogue of isonemal fabrics', in Discrete Geometry and Convexity, Editors J.E. Goodman et al., pp. 279-298 (Annals New York Acad. Sci. 440, 1985).

[7] B. Grünbaum and G.C. Shephard, 'Isonemal fabrics', Amer. Math. Monthly 95 (1988), 5-30.

[8] B. Grünbaum and G.C. Shephard, 'Satins and twills', Math. Mag. 53 (1980), 139-161.

[9] B. Grünbaum and G.C. Shephard, Tilings and patterns (Freeman, New York, 1986).

[10] W.D. Hoskins and R.S.D. Thomas, 'Conditions for isonemal arrays on a cartesian grid', Linear Algebra Appl. 57 (1984), 87-103.

[11] J.A. Hoskins, R.G. Stanton and A.P. Street, 'Enumerating the compound twillins', Congr. Numer. 38 (1983), 3-22.

[12] J.R. Lourie, 'Loom-constrained designs: An algebraic solution', Proceedings of the ACM National Conference (1969), 185-192.

[13] D. Schattschneider, 'M.C. Escher's classification system for his colored periodic drawings', in M.C. Escher: Art and Science, Editors H.S.M. Coxeter, pp. 82-96 (North-Holland, Amsterdam, 1986).

Department of Computer Science

University of Manitoba

Winnipeg, Manitoba

Canada R3T 2N2

\author{
Department of Applied Mathematics \\ University of Manitoba \\ Winnipeg, Manitoba \\ Canada R3T 2N2
}

